\title{
Study on knowledge, attitude and dog ownership patterns related to rabies prevention and control in Addis Ababa, Ethiopia
}

\author{
${ }^{1^{*}}$ Eshetu Yimer, Arthuro Mesfin, ${ }^{2}$ Mekoro Beyene, ${ }^{2}$ Abebe Bekele, ${ }^{2}$ Girum Taye, Badeg \\ Zewdie, and ${ }^{3}$ Tsega Alemayehu \\ ${ }^{1}$ Veterinary Consultant, \\ ${ }^{2}$ Ethiopian Health and Nutrition Research Institute, P. O. Box 1242/5654, \\ ${ }^{3}$ Addis Ababa Region Agricultural Bureau \\ *Corresponding author: Eshetu Yimer,e-mailesyima_n@yahoo.com
}

\begin{abstract}
The study was conducted from May 2003 to August 2003 in Addis Ababa with the objective of understanding the distribution of stray and owned dogs, dog ownership patterns and attitudes of people towards rabies and its prevention and control methods. A total of 2390 households were selected from 6 Sub Cities of Addis Ababa using stratified random sampling and were interviewed using structured questionnaires. From the total households interviewed, 969 (40.5\%) of them were known to own one or more dogs, and the total number of owned dogs was estimated to be 225,078. Male dogs make up 1042 (78.4\%) of the total owned dogs. Almost half of all the dogs 463 (47.8\%) were tied only for some time during the day whereas $320(33.1 \%)$ of the dogs were not tied at all and freely move from place to place and contribute to high dog bites in human beings and for the widespread occurrence of canine rabies in Addis Ababa. Six hundred fifty five (67.6\%) of the interviewed households who owned dogs had a well fenced house. Most of the owned dogs $856(88.4 \%)$ get their food from their owners and only $321(33.3 \%)$ of the owners reported that they let their dogs to be vaccinated while the majority of the owners $644(66.5 \%)$ do not let their dogs vaccinated regularly. A significant proportion of the interviewed households 2,323 (97.2\%), $(\mathrm{P}<0.05)$ indicated that they have the knowledge that dogs, cats and other animals can transmit rabies to humans and $1,752(73.4 \%)$ of the households replied that rabies can be transmitted through bite, scratch and lick to open wounds. Analysis of the list of 2400 post exposure human antirabies treatments recorded at the EHNRI Zoonoses Laboratory from February 2002 to October 2003 showed that the most common animals involved in biting people were dogs. A total of 2198 (91.6\%) people were bitten by dogs during
\end{abstract}


the same period and most of them, 2053 (85.5\%) were bitten on their hands and legs. All the above data indicated the need for a strong and coordinated rabies control activities to be conducted in the city.

Keywords: Addis Ababa, Attitude, Dog ownership, Knowledge, Rabies

http://dx.doi.org/10.4314/evj.v16i2.3

\section{Introduction}

Rabies is one of the oldest known and invariably fatal diseases once clinical signs develop (Bernard \& Hattwick, 1985). Rabies is caused by a Rhabdovirus and is transmitted to animals or human through bite by rabid animals. In Ethiopia it is primarily a disease of dogs that has been recognized for many centuries (Fekadu 1982).

Data recorded at the Ethiopian Health and Nutrition Research Institute (EHNRI) rabies diagnostic unit showed that zones 2, 3, 4 and 5 under the previous administration structure, were recorded as having the highest incidences of dog bite during the last six months of 2001. Despite the lack of sufficient information to scale the magnitude of rabies in dogs and humans in the country as a whole, during the period 1990-2000, dogs contributed to $95 \%$ of the total fatal human rabies cases and $96 \%$ of the total animals examined after being incriminated in biting of humans (Eshetu et al 2002). During the year 2000, a total of 1540 people received post exposure anti-rabies treatment in Addis Ababa and its surroundings of which 1443 (93.70\%) were due to dog bites. Hence, dogs are considered as the major biting animals and most important sources of the disease to humans in Ethiopia.

As indicated by Bögel, (1984) any rabies control initiative should aim at controlling the disease in dogs. Hence, inventory of dog population in a community is very essential to know the approximate number of dogs and design appropriate vaccination schedules and also assists to control stray dogs in order to effectively prevent and control the disease. Therefore this study was designed to generate preliminary information on dog population, dog ownership patterns and socioeconomic status of the community in relation to dog ownership, rabies situation and rabies control in Addis Ababa. 


\section{Materials and Methods}

\section{Study Area}

This study was carried out in Addis Ababa, which covers an area of $540 \mathrm{~km}^{2}$ and had 10 Sub Cities and 203 villages (kebelles) as an administrative region. The city lies at an altitude between 2300-2500 meters above sea level with an average temperature ranging between $8.9^{\circ} \mathrm{C}-24.42^{\circ} \mathrm{C}$. During the study period, the total human population of Addis Ababa was estimated to be 3 million (Statistical abstracts 2002).

\section{Sampling Design}

At the time of the study Addis Ababa was divided into three divisions; Inner city, that encompasses previous woredas 1-7, Old inner city previous woredas 8-16, and Outer city previous woredas 17-28. For this study, 6 sub cities were selected that included two from each of the three divisions. Arada and Addis Ketema sub cities from Inner city, Yeka and Bole from Old inner city and Akaki and Kolfe Keranio sub cities from Outer city were selected. Stratified random sampling was utilized to select the study households. From each division 2 sub cities, and from each sub city 1 kebelle are taken as a strata and 200 households from each kebele were selected by systematic random sampling and households were interviewed using a structured questionnaire developed for the study. Key informants were selected from each kebelle and focused group discussion (FGD) was also held.

Human post exposure treatment records $(n=2400)$ at EHNRI was analyzed to determine the nature of bite and the condition and fate of the biting animal. Survey of stray dogs was conducted at night in selected areas. In addition a day time estimate was recorded based on the information obtained from the respondents. SPSS statistical software was utilized for data entry and analysis. Chi- Square $\left(\mathrm{X}^{2}\right)$ test was used to calculate significant differences among proportions of variables.

\section{Results}

The study revealed that 969 (40.5\%) of the households interviewed were found to own one or more dogs from which the total number of owned dog population in the capital was estimated to be 225078. Male dogs make up 1042 (78.4\%) of the total owned dogs. 
Almost half of all the dogs $463(47.8 \%)$ (Table 1) were tied only for some time during the day whereas $320(33.1 \%)$ of the dogs were not tied at all. Some respondents reported as having seen as high as 100 stray dogs in their surroundings.

Six hundred fifty five (67.6\%) of the interviewed households who owned dogs had a well fenced houses. Owned dogs which accounted 856 (88.4\%) get their food from their owners and of these, 321 (33.3\%) of them received vaccination regularly. A significant proportion of the interviewed households 2323 (97.2\%), $(\mathrm{P}<0.05)$ indicated that dogs, cats and other animals do transmit rabies to humans and 1752 (73.4\%) of the households replied that biting, scratching and licking transmit rabies.

Of the household interviewee 2,053 (85.9\%), $(\mathrm{P}<0.05)$ indicated that wound treatment and receiving post exposure vaccination would prevent acquiring rabies when there is sudden bite by rabid or suspected rabid dogs and cats. Analysis of the educational status of the interviewee revealed that $688(28.8 \%)$ of the dog owners took elementary education and 823 (34.4\%) had no education at all.

Orthodox Christians, Protestants and Catholics and others that make up 944 (97.4\%) (Table 2 ) of the interviewee owned dogs while only 25 (2.6\%) of Muslims keep dogs. During the focused group discussion, the participants indicated that rabies is a major problem in Addis Ababa, there are large number of uncontrolled dogs, the absence of regular vaccination of dogs and stray dog elimination that result in many people to be bitten by rabid or suspected rabid dogs.

Analysis of the list of 2400 post exposure human antirabies treatments recorded at the EHNRI Zoonoses Laboratory from February 2002 to October 2003 showed that the most common animals involved in biting people were dogs. To that effect a total of 2198 


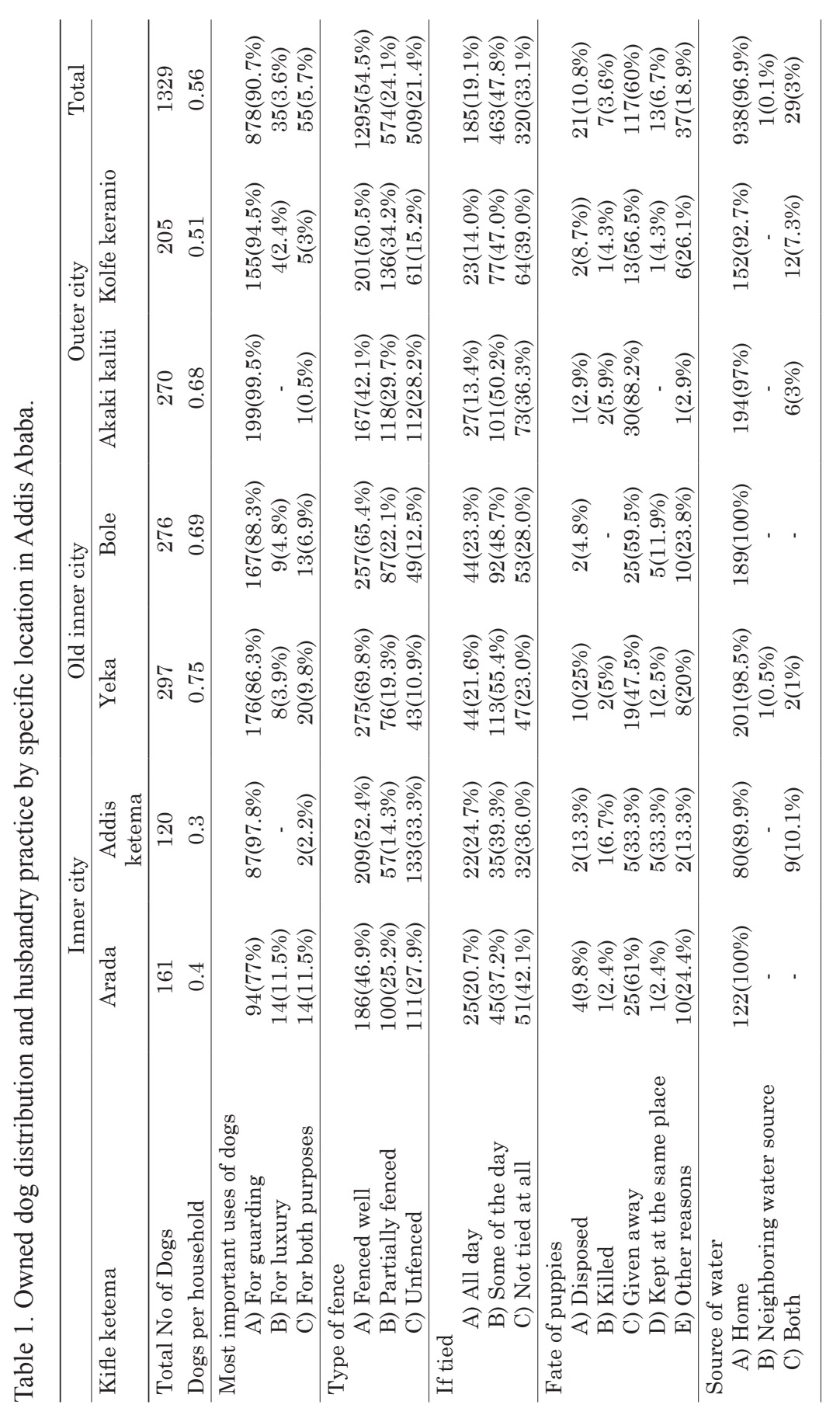




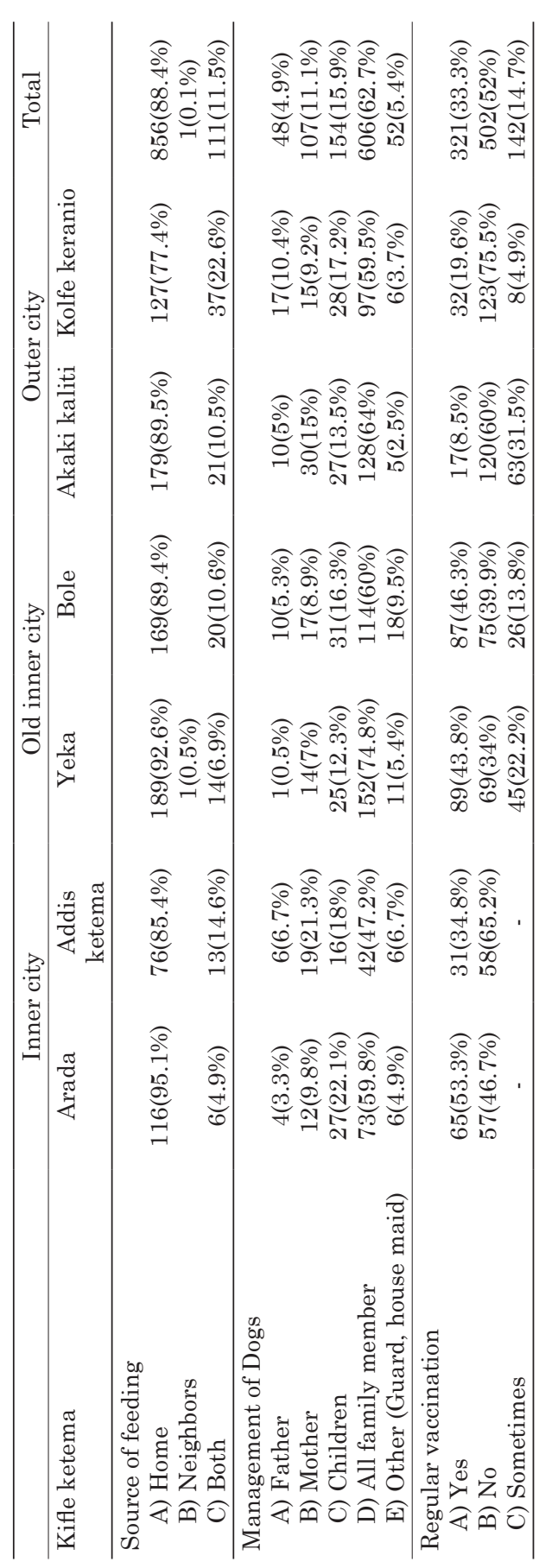




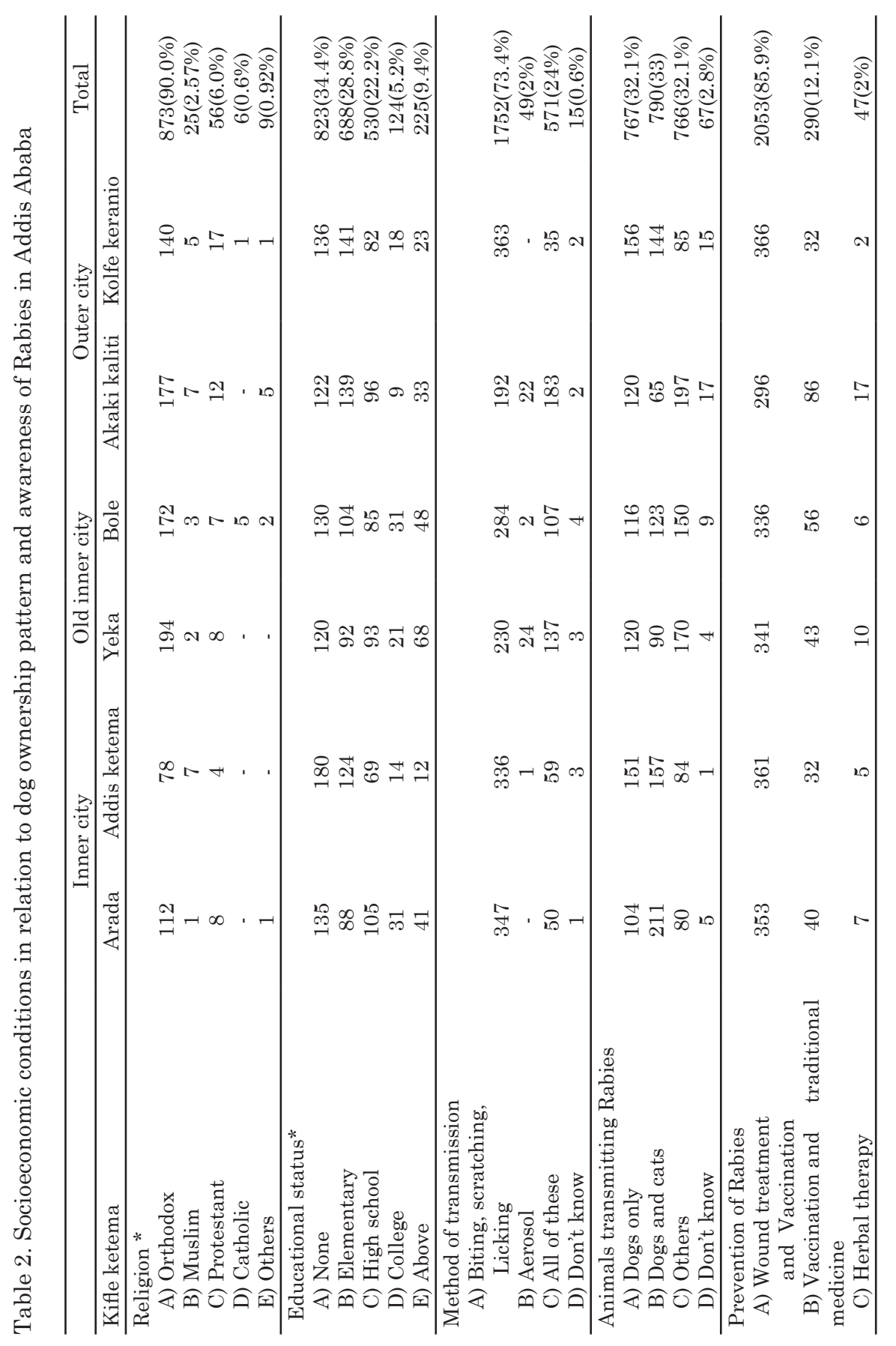

Ethiop. Vet. J., 2012, 17 (2), 27-39 
(91.6\%) people were bitten by dogs during the same period and most of them, 2053 (85.5\%) were bitten on their hands and legs. In addition, the data recorded also showed that 943 (39.3\%) of the dog bit victims that sought the treatment were children under 15 years of age and the post exposure antirabies treatment was given to 1159 (53.2\%) people bitten by dogs that bite and escaped,

\section{Discussion}

This study revealed that of the total 2390 households interviewed 969 (40.5\%) of the households were found to own one or more dogs and the total number of owned dog population in the capital was estimated to be 225078. This finding was higher than the estimate for owned dogs given some 25 years ago (Fekadu, 1982). Some respondents reported as having seen as high as 100 stray dogs in their surroundings, the exaggerated figure reported and the possibility of observing the same dog repeatedly made it very difficult to estimate the total number of stray dogs found in the capital.

Fences are very important in restricting the movement of dogs out side of their residences and also prevent contact between dogs. One hundred eighty five (19.1\% ) of the owners that keep their dogs tied during the whole day were well aware of the dangers associated with exposure to rabies due to free movement of dogs outside their compounds. However, only 655 (67.6\%) of the households keep their dogs in well-fenced houses. Those untied dogs freely move from place to place and contribute to the transmission of the disease and widespread occurrence of canine rabies in Addis Ababa. It is advisable to keep dogs in well fenced compounds and get them vaccinate them against rabies. Sometimes uncontrolled cats when become rabid, could easily jump over fences and houses and attack tied dogs inside their compounds and thus transmit the disease to healthy unvaccinated dogs. The fact that $320(33.1 \%)$ of the owned dogs are not tied at all may contribute to the widespread nature of rabies in the capital due to increased contact between susceptible and rabid dogs.

All dogs at home should be provided with adequate food every day. If dogs are not fed adequately and given proper shelter, then they may run away and roam around in search of food thus becoming easily attacked by rabid dogs. Later when they come back home again they can pose a serious danger of transmitting the disease to their owners who are not well aware of the contact that their dogs had with other rabid dogs. The above condition emphasizes the fact that as long as dogs are kept for the benefit that they provide in guarding home in most cases in our country, they should also be given adequate care and 
protection. Dogs at home should be properly handled and fed with adequate provision of medical care including regular vaccinations in order to protect them from rabies and ultimately prevent the family and also the communities against rabies.

This study showed that only 321 (33.3\%) of the dog owners interviewed vaccinate their dogs against rabies regularly while the larger majority of the owners do not vaccinate their dogs. Therefore, dog owners should be advised to get their pets vaccinated regularly so that the occurrence of the disease in pets and ultimately in humans would be minimized. Observation in relation to dogs ownership based on educational level showed that 534 (55.1\%) of the dog owners did have elementary or no education at all which may have a direct influence on the care and handling of dogs and fulfilling of their requirements in terms of food, shelter, medical care including regular vaccinations by these owners.

Among the interviewee, Orthodox Christians Protestants and Catholics make up 944 (97.4\%) of the dog owning community and dogs are mainly utilized by them for guarding and luxury while only 25 (2.6\%) of the Muslims keep dogs for guarding because of their religious principle that prohibits handling and owning dogs.

The focused group discussion that was held with selected key informants from each kebele and the Kebele officials clearly demonstrated that rabies is a serious problem in Addis Ababa. The presence of large number of uncontrolled dogs was strongly stressed and that an immediate integrated action was suggested to be undertaken to control and prevent the occurrence of the disease in Addis Ababa.

Rabies is a well established disease in Ethiopia and most of the respondents are well aware of the risks associated with the disease, the importance of modern human post exposure treatment (vaccination) and proper wound treatments. A significant proportion of the interviewed households 2323 (97.2\%), $(\mathrm{P}<0.05)$ indicated that rabies is transmitted to humans when they are bitten, scratched or licked by rabid dogs, cats and other animals. In addition, most of the households $2053(85.9 \%)$ indicated that treatment of wound and vaccination is important to prevent the occurrence of rabies in humans when bitten by suspected or known rabid dogs and cats. This showed that the disease is long known in the country and the existence of some awareness about the disease.

Ethiop. Vet. J., 2012, 17 (2), 27-39 
Yilma Ayalew (1984) indicated that the wide spread use of supposed traditional anti rabies herbal remedies were aggravating mortality due to rabies. This is because most of the affected people were utilizing these remedies. In some of the cases those who started even vaccinations were persuaded locally by their relatives to disrupt the vaccination regime and start these herbal remedies which are without effective results. Contrary to the above finding, $85.7 \%$ of the interviewed households in this study indicated the treatment of wound and vaccination of people bitten by rabid or suspected rabid animals mainly dogs as the basis for the prevention of the occurrence of the disease in humans. This may be directly associated with the availability of modern treatment in the capital. However in most rural communities although rabies is a well known disease, the treatment option most commonly available to the people in these remote areas is herbal therapy with unknown efficacy and mostly non effective in preventing the development of the disease in humans.

The human post exposure antirabies treatment data recorded at zoonoses laboratory of EHNRI showed that 943 (39.3\%) of the dog bit victims that sought the treatment were children under 15 years of age. This indicated the need for more attention to be given in protecting the helpless children to avoid the most arduous and stressful treatment schedules.

Post exposure antirabies treatment was given to 1159 (53.2\%) people bitten by dogs that bite and escaped, as indicated by the study participants this could be associated with the presence of large number of stray dogs in the streets of Addis Ababa. This showed that people in the capital have the risk of being attacked and developing rabies by free roaming dogs unless they get early medical treatment. Hence controlling free roaming dogs in the capital requires a very serious attention.

As documented historical evidence in Addis Ababa in 1941, the number of people who sought treatment every month because of bite by rabid dogs was about 100 that increased to more than 3000 in 1956 (Schaller, 1972). Annually an average of about 5230 people received post exposure anti rabies treatment all over the country during the period $1964-1975$ (Fekadu 1982). Compared to the above information, an average of about 6 persons receive post exposure anti rabies treatments daily in Addis Ababa (EHNRI) alone during the last 15 years period of $1990-2004$. This showed that people seeking post exposure antirabies treatment is on the increase. 
This study estimated the total population of owned dogs in Addis Ababa to be 225,078 . This may be an underestimate of the actual population of owned dogs as it only considers only four months of the year. Therefore, a wider scale and year round study may be required to estimate the total population so that appropriate dog and rabies control strategies could be designed. This study complements previous findings on the significance of dogs as the major source of human infection and rabies as one of the most important zoonotic diseases that posed serious public health hazard for centuries in Ethiopia.

Under the Ethiopian condition it is even very difficult to clearly define dog ownership pattern due to the fact that strictly confined dogs are very rare and most dogs claimed owned are free to move around outside of their compound for most of the day. A dog that freely roams around is considered as a stray dog.

Addis Ababa when it had a human population of about 1 million, was estimated to have 150,000 - 200,000 stray dogs some 25 years ago (Makonnen Fekadu 1982). At the moment with an estimated human population of about 3 million, the number of stray dogs is expected to be correspondingly higher. Therefore, as indicated by Bogel (1984) any rabies control initiative should aim at controlling the disease in dogs. Canine rabies has been successfully controlled in many European countries as evidenced by the rarity of human rabies cases in the developed world while it is still a big challenge in the developing countries (Debbie 1988).

\section{Conclusion and recommendations}

From the current study it can be concluded that there are many dogs which are owned but not restricted by much of the households included in the study and the reason of keeping dogs is for safeguarding and companionship. Ownership to dogs is very poor that feeding, housing and health care by the owners is not fully practiced. The study showed that there is knowledge of the risk of rabies transmission through dogs' bites and rabies prevention measures upon human exposure. However, dog vaccination is poorly practiced.

Rabies is prevalent in Addis Ababa and the sources of infection to humans are dogs and most post exposure antirabies treatments given to humans are primarily to dog bites, but also the number of stray dogs in Addis Ababa is very large and those owned are not tied for the whole day. Christians keep dogs more than the Muslims in Addis Ababa. There is no regular vaccination 
of dogs and cats in Addis Ababa and most inhabitants are aware of the dangers associated with rabies.

Therefore based on the above findings the following recommendations are forwarded.

There must be legislation with adequate enforcement requiring the registration, licensing and taxation of dogs, a measure which is often considered as the basis for mass immunization and dog population control.

Regular vaccination campaigns should be practiced and this should be combined with continuing vaccination scheme for young dogs. Vaccinated dogs must be marked in order to facilitate removal of non-vaccinated animals. The capture and impoundment of stray dogs should be reinforced. Adequate rabies diagnostic facilities should be available and placed at central places to serve utilizing areas. Suspected animals should be quarantined for 10 days (WHO, 1996). Dogs that show clinical signs or have been in close contact or bitten by a rabid or suspected rabid animal should be destroyed.

Although there is a certain level of awareness about rabies in the public, further public education are still essential. Posters, newspapers, radio and television announcements should be used for health education to create awareness among the community. The major task of education should focus on first aid treatments that should be sought immediately after bite and also on inducing a national sense of purpose to teaching communities that dog bites should be washed immediately, even if no soap or vaccine is available.

Addis Ababa being the Capital of Africa, there is an urgent need for a coordinated rabies control activities by forming a standing committee or any other relevant body. A successful pilot rabies control programme in Addis Ababa could serve as a model to cover nearby towns and other urban and rural areas in Ethiopia.

\section{Acknowledgments}

The authors would like to express their thanks to the Ethiopian Science and Technology Commission (ESTC) for funding this project and the Ethiopian Health and Nutrition Research Institute for the administrative and technical supports. 


\section{References}

Abebe, P., Yimer E., Newayeselassie, B., Bekele, A. Zewdie, B., and Beyene, M 2003. A Study on the Prevalence of Animal Rabies in Addis Ababa Ethiop. V. Journal 7 (1\&2), pp 69-77.

Ayalew, Y. 1985. Analysis of 159 human rabies cases in Ethiopia In: Kuwert E, Meirieux C. pp 392-294.

Bernard, K.W. and Hattwick, M.A.W. 1985. Rhabdoviridae; Rabies virus In: principles and Practice of Infectious Diseases Eds; Mandell G.L., Douglas R.G. and Bennett J.E. $2^{\text {nd }}$ ed. P. $897-909$.

Bogel, K. 1984 Guidelines for dog rabies control, WHO, VPH/83.43

Debbie, J.G.1988 Rabies: an old enemy that can be defeated World Health Forum,WHO vol.9 No.4:536 -541.

Fekadu, M. 1982. Rabies in Ethiopia. Am. J. Epidemiol. 115: 266 - 73.

Fekadu, M. 1997. Human rabies surveillance and control in Ethiopia, In: Proceedings of the Southern and Eastern Africa Rabies Group Meeting Nairobi, Kenya 4-6 March 1997.

RABNET. 1998. Survey of Rabies for the year 1998 No 34 WHO Department of Communicable Disease Surveillance and Response WHO/CDS/CSR/APH/99.6

Schaller K.F. 1972. Ethiopia. A Geomedical Monograph Springer-Verlag Berlin Heidelberg, New York p27

Statistical Abstracts 2002. FDRE Office of Population and Housing Census Commission. The 1994 Population and Housing Census of Ethiopia Results at COUNTRY LEVEL Vol. 1 STATISTICAL REPORT.

WHO. 1996. WHO Recommendations on Rabies Post-Exposure Treatment and the Correct Technique of Intradermal Immunization against Rabies.

Yimer, E., Newayeselassie, B., Tefera, G., Mekonnen, Y., Bogale, Y., Zewdie, B., Beyene, M. and Bekele, A. 2002. Situation of Rabies in Ethiopia: A retrospective study from 1990-2000. Ethiop. J. Health Development 16(1): 105-112. 
